\title{
BCS-BEC crossover of neutron pairs in symmetric and asymmetric nuclear matter
}

\author{
J. Margueron, ${ }^{1,2}$ H. Sagawa, ${ }^{2}$ and K. Hagino ${ }^{3}$ \\ ${ }^{1}$ Institut de Physique Nucléaire, Université Paris-Sud, IN $P_{3}-C N R S$, F-91406 Orsay Cedex, France \\ ${ }^{2}$ Center for Mathematical Sciences, University of Aizu, Aizu-Wakamatsu, 965-8580 Fukushima, Japan \\ ${ }^{3}$ Department of Physics, Tohoku University, Sendai, e980-8578, Japan
}

(Dated: November 5, 2018)

\begin{abstract}
We propose new types of density dependent contact pairing interaction which reproduce the pairing gaps in symmetric and neutron matter obtained by a microscopic treatment based on the nucleon-nucleon interaction. These interactions are able to simulate the pairing gaps of either the bare interaction or the interaction screened by the medium polarization effects. It is shown that the medium polarization effects cannot be cast into the density power law function usually introduced together with the contact interaction and require the introduction of another isoscalar term. The BCS-BEC crossover of neutrons pairs in symmetric and asymmetric nuclear matter is studied by using these contact interactions. It is shown that the bare and screened pairing interactions lead to different features of the BCS-BEC crossover in symmetric nuclear matter. For the screened pairing interaction, a two-neutron BEC state is formed in symmetric matter at $k_{F n} \sim 0.2 \mathrm{fm}^{-1}$ (neutron density $\left.\rho_{n} / \rho_{0} \sim 10^{-3}\right)$. Contrary the bare interaction does not form the BEC state at any neutron density.
\end{abstract}

PACS numbers: 21.30.Fe, 21.60.-n, 21.65.+f, 25.70.-z, 26.60.+c

Keywords: effective density-dependent pairing interactions, symmetric and asymmetric nuclear matter, BCSBEC crossover.

\section{INTRODUCTION}

Low density nuclear matter tends to be much more interesting than a simple zero density limit of the bulk physics of nuclei. Indeed, new phenomena show up and are mainly associated with the formation of bound states or with the emergence of strong correlations 1, 2, 3, 4, 5, 6]. For instance, in symmetric nuclear matter, neutrons and protons become strongly correlated while the density decreases and a deuteron BEC state appears at very low density [7]. The deuteron-type correlations give extra binding to the nuclear equation of state and induce new features at low density 8]. This transition belongs to the BCS-BEC crossover phenomena which have been extensively studied in several domains of physics and has recently been experimentally accessible in cold atomic gas (see Ref. [9] and references therein).

In nuclear matter, neutron pairs are also strongly correlated. Theoretical predictions suggest that, at density around $\rho_{0} / 10$ where $\rho_{0}=0.16 \mathrm{fm}^{-3}$, the ${ }^{1} \mathrm{~S}$ pairing gap may take a considerably larger value than that at normal nuclear density $\rho_{0}$ 10]. The density dependence of the pairing gap at low density is unfortunately not yet completely clarified and still awaits a satisfactory solution [11, 12, 13]. Therefore, it could be interesting to explore pairing interactions based on Brueckner theory and its consequences to the BCS-BEC crossover. Indeed, pairing at low density is relevant for different purposes: for the understanding of neutron-rich exotic nuclei near the drip line 14, 15, 16, 17, 18, 19], where the long tails of density profiles give rise to "halo" or "skin" behavior, or for the expanding nuclear matter in heavy ion collisions [20], or even for the physics of neutron stars, where several physical phenomena, such as cooling and glitches, are thought to depend very sensitively on the size and the density dependence of the pairing gap [21, 22, 23]. In ${ }^{11} \mathrm{Li}$, the wave function of the two neutrons participating to the halo nucleus has been analyzed with respect to the BCS-BEC crossover 24]. It has been shown that as the distance between the center of mass of the two neutrons and the core increases, the wave function changes from the weak coupling BCS regime to the strongly correlated BEC regime. This is due to the fact that the pairing correlations are strongly density-dependent [25] and the distance between the two neutrons and the core provides a measure of the pairing strength.

It should be emphasized that the bare nuclear interaction in the particle-particle channel should be corrected by the medium polarization effects [10, 11] (usually referred to as the screening effects). These effects have been neglected for a long time since the nuclear interaction is already attractive in the ${ }^{1} \mathrm{~S}_{0}$ channel without the medium polarization effects, contrary to the Coulomb interaction for which the medium polarization effects are absolutely necessary to get an attractive interaction between electrons. However, several many-body methods have been developed recently to include the medium polarization effects in the calculation of the pairing gap such as a group renormalization method [26], Monte-Carlo methods calculation 27, 28, 29] and extensions of the Brueckner theory 10, 11]. These calculations, except the one presented in Ref. [27], predict a reduction of the pairing gap in neutron matter.

Note that, based on the nuclear field model, it has also been suggested that the medium polarization effects contribute to the pairing interaction in finite nuclei and increase the pairing gap [30, 31, 32]. To understand this apparent contradiction between neutron matter and finite nuclei, a Brueckner calculation including the medium po- 
larization effects in both symmetric and neutron matter has been performed in Ref. [11]. It has been shown that the medium polarization effects are different in neutron matter and in symmetric matter. The medium polarization effects do not reduce the pairing gap in symmetric matter, contrary to that in neutron matter. Instead, in symmetric matter, the neutron pairing gap is much enlarged at low density compared to that of the bare calculation. This enhancement takes place especially for neutron Fermi momenta $k_{F n}<0.7 \mathrm{fm}^{-1}$. This could explain why the medium polarization effects increase largely the pairing correlations in finite nuclei but decrease it in neutron matter.

In this paper, we propose an effective densitydependent pairing interaction which reproduces both the neutron-neutron (nn) scattering length at zero density and the neutron pairing gap in uniform matter obtained by a microscopic treatment based on the nucleon-nucleon interaction [11]. The proposed interaction has isoscalar and isovector terms which could simultaneously describe the density dependence of the neutron pairing gap for both symmetric and neutron matter. Furthermore, we invent different density-dependent interactions to describe the "bare" and "screened" pairing gaps, together with the asymmetry of uniform matter, given in Ref. [11]. Then, we explore the BCS-BEC crossover phenomena in symmetric and asymmetric nuclear matter.

This paper is organized as follows. In Sec. III we discuss how to determine the isoscalar and isovector densitydependent contact interactions. Applications of those interactions to the BCS-BEC crossover are presented in Sec. III. We give our conclusions in Sec. IV]

\section{DENSITY-DEPENDENT PAIRING INTERACTION}

Recently, spatial structure of neutron Cooper pair in low density nuclear matter has been studied using both finite range interactions like Gogny or G3RS and density-dependent contact interactions properly adjusted to mimic the pairing gap obtained with the former interactions 25]. It was found that the contact interactions provide almost equivalent results compared to the finite range ones for many properties of the Cooper pair wave functions. It is thus reasonable to investigate the evolution of the Cooper pair wave function with respect to the density and the isospin asymmetry using contact interactions adjusted to realistic interactions. In this paper, we take a contact interaction $v_{n n}$ acting on the singlet ${ }^{1} \mathrm{~S}$ channel,

$$
\left\langle k\left|v_{n n}\right| k^{\prime}\right\rangle=\frac{1-P_{\sigma}}{2} v_{0} g\left[\rho_{n}, \rho_{p}\right] \theta\left(k, k^{\prime}\right),
$$

where the cutoff function $\theta\left(k, k^{\prime}\right)$ is introduced to remove the ultraviolet divergence in the particle-particle channel. A simple regularization could be done by introducing a cutoff momentum $k_{c}$. That is, $\theta\left(k, k^{\prime}\right)=1$ if $k, k^{\prime}<k_{c}$ and 0 otherwise. In finite systems, a cutoff energy $e_{c}$ is usually introduced instead of a cutoff momentum $k_{c}$. The relation between the cutoff energy and the cutoff momentum may depend on the physical problem, and it is known that the pairing strength $v_{0}$ depends strongly on the cutoff. A detailed discussion on the different prescriptions used in the literature are then presented in Appendix A In this paper, we choose the prescription 3 in the Appendix $\AA$ so that the adjusted interaction can be directly applied to Hartree-Fock-Bogoliubov calculations.

In Eq. (11), the interaction strength $v_{0}$ is determined by the low energy scattering phase-shift, that fixes the relation between $v_{0}$ and the cutoff energy $e_{c}$, while the density-dependent term $g\left[\rho_{n}, \rho_{p}\right]$ is deduced from predictions of the pairing gaps in symmetric and neutron matter based on the nucleon-nucleon interaction [11]. The density-dependent term accounts for the medium effects and satisfies the boundary condition $g \rightarrow 1$ for $\rho \rightarrow 0$. The volume type and surface type pairing interactions have $g=1$ and $g=0$ at $\rho=\rho_{0}$, respectively. In this paper, we introduce more general types of pairing interactions and the novelty is a dependence on the ratio of neutron to proton composition of the considered system. We thus define a function

$$
g_{1}\left[\rho_{n}, \rho_{p}\right]=1-f_{s}(I) \eta_{s}\left(\frac{\rho}{\rho_{0}}\right)^{\alpha_{s}}-f_{n}(I) \eta_{n}\left(\frac{\rho}{\rho_{0}}\right)^{\alpha_{n}},
$$

where $I$ is the asymmetry parameter, defined as $I=$ $(N-Z) /(N+Z)$, and $\rho_{0}=0.16 \mathrm{fm}^{-3}$ is the saturation density of symmetric nuclear matter. We insert the function $g_{1}$ into Eq. (11) as $g=g_{1}$. The goal of the functional form in Eq. (2) is to reproduce the theoretical calculation of the pairing gap in both symmetric and neutron matter and also to be used for prediction of the pairing gap in asymmetric matter. It could also be applied to describe pairing correlations in finite nuclei by acquiring an explicit dependence on the coordinate $r$ in the density $\rho(r)$ and the asymmetry parameter $I(r)$. In Eq. (2), the interpolation functions $f_{s}(I)$ and $f_{n}(I)$ are not explicitly known but should satisfy the following condition $f_{s}(0)=f_{n}(1)=1$ and $f_{s}(1)=f_{n}(0)=0$. The densitydependent function $g_{1}$ is flexible enough and we can obtain an effective pairing interaction which reproduces the density dependence of the pairing gap in uniform matter. It should however be noticed that the interpolation functions $f_{s}(I)$ and $f_{n}(I)$ cannot be deduced from the adjustment of the pairing gap in symmetric and neutron matter. For that, theoretical calculations in asymmetric nuclear matter or application to exotic nuclei are necessary. In this paper, we choose $f_{s}(I)=1-f_{n}(I)$ and $f_{n}(I)=I$. Many different interpolation functions could be explored but we think that it has little consequences to the BCS-BEC crossover.

We have also explored other density-dependent functionals, introducing an explicit dependence on the isovector density, $1-\eta_{s}\left(\frac{\rho}{\rho_{0}}\right)^{\alpha_{s}}-\eta_{i}\left(\frac{\rho_{n}-\rho_{p}}{\rho_{0}}\right)^{\alpha_{i}}$, or introducing the neutron and proton densities, $1-\eta_{n}\left(\frac{\rho_{n}}{\rho_{0}}\right)^{\alpha_{n}}-$ 

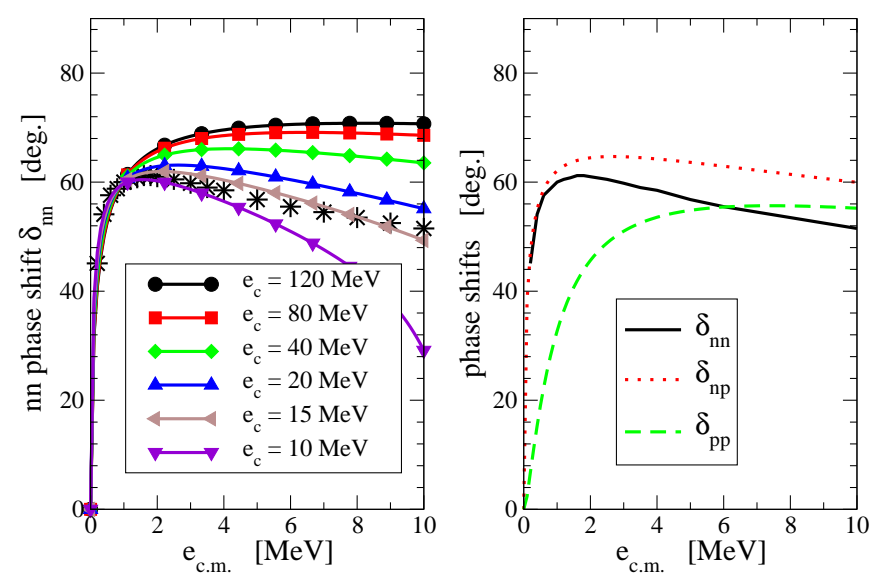

FIG. 1: (Color online) Phase shifts for s-wave nucleonnucleon scattering as a function of the center of mass energy. In the left panel are shown nn phase shifts obtained from Argonne $v_{18}$ potential [16] (stars) and the result of the best adjustment obtained with a contact interaction for a set of cutoff energies (from 10 to $120 \mathrm{MeV}$ ). In the right panel are shown the s-wave phase shifts for various channels: $n n, n p$ and $\mathrm{pp}$. The $\mathrm{np}$ and $\mathrm{pp}$ phase shifts have been provided by the Nijmegen group (http://nn-online.org).

$\eta_{p}\left(\frac{\rho_{p}}{\rho_{0}}\right)^{\alpha_{p}}$. The isospin dependence of those functionals is fixed but these functional forms are not flexible enough so that the adjustment becomes sometimes impossible. For instance, the pairing gap in symmetric and neutron matter including the medium polarization effects and calculated in Ref. [11] cannot be reproduced with such functionals. In the following, we have therefore considered only the density-dependent interaction given in Eq. (2).

Recently, another attempt has been done to introduce an isospin dependent volume type pairing interaction in a very different approach [34]. This interaction has been adjusted to reproduce empirical mass formula over a thousands of nuclei, but the pairing gap calculated with this pairing interaction compares very badly with realistic calculations in uniform matter.

\section{A. The free interaction}

In Ref. [14], it has been proposed to deduce the free interaction parameter $v_{0}$ from the low energy phase shift of nucleon-nucleon scattering. The nn, np and pp phase shifts versus the center of mass energy are shown on the right panel of Fig. 1, It is clear from this figure that each of these three channels are different and the interaction parameter $v_{0}$ should depend on the channel of interest. In this paper, we are interested only in the nn channel. We then express the phase shift as a function of the cutoff

\begin{tabular}{ccccccc}
\hline \hline $\begin{array}{c}e_{c} \\
{[\mathrm{MeV}]}\end{array}$ & $\begin{array}{c}a_{n n} \\
{[\mathrm{fm}]}\end{array}$ & $\begin{array}{c}r_{n n} \\
{[\mathrm{fm}]}\end{array}$ & $\begin{array}{c}\alpha \\
{[\mathrm{fm}]}\end{array}$ & $\begin{array}{c}v_{0} \\
{\left[\mathrm{MeV} . \mathrm{fm}^{3}\right]}\end{array}$ & $\begin{array}{c}v_{0}^{*} \\
{\left[\mathrm{MeV} \cdot \mathrm{fm}^{3}\right]}\end{array}$ & $\begin{array}{c}v_{0}^{\infty} \\
{\left[\mathrm{MeV} \cdot \mathrm{fm}^{3}\right]}\end{array}$ \\
\hline 120 & -12.6 & 0.75 & -0.55 & -448 & -458 & -481 \\
80 & -13.0 & 0.92 & -0.66 & -542 & -555 & -589 \\
40 & -13.7 & 1.30 & -0.91 & -746 & -767 & -833 \\
20 & -15.0 & 1.83 & -1.25 & -1024 & -1050 & -1178 \\
15 & -15.7 & 2.12 & -1.43 & -1167 & -1192 & -1360 \\
10 & -17.1 & 2.59 & -1.72 & -1404 & -1421 & -1666 \\
\hline \hline
\end{tabular}

TABLE I: For a given cutoff energy $e_{c}$, the parameters $r_{n n}$, $\alpha$ and $v_{0}$ are determined by the scattering length $a_{n n}$ which reproduces the phase shift in the low energy region $e_{c . m}$. $<$ $2 \mathrm{MeV}$. The interaction strengths $v_{0}^{*}$ and $v_{0}^{\infty}$ are obtained from the empirical value of $a_{n n}=-18.5 \mathrm{fm}$ and from the unitary limit, defined as $a_{n n} \rightarrow \infty$.

momentum $k_{c}$ and the scattering length $a_{n n}$ [16, 33]:

$$
\begin{aligned}
k \cot \delta & =-\frac{2}{\alpha \pi}\left[1+\alpha k_{c}+\frac{\alpha k}{2} \ln \frac{k_{c}-k}{k_{c}+k}\right] \\
& =-\frac{1}{a_{n n}}-\frac{k}{\pi} \ln \frac{k_{c}-k}{k_{c}+k}
\end{aligned}
$$

where $\alpha=2 a_{n n} /\left(\pi-2 k_{c} a_{n n}\right)$. In this way, for a given cutoff momentum $k_{c}$, the phase shift can be adjusted using the scattering length $a_{n n}$ as a variable. The results are shown in Fig. 1 and Table 1 for a set of cutoff energies $e_{c}=\hbar^{2} k_{c}^{2} / m$ (note that we use the reduced mass $m / 2$ ). We have found that, for cutoff energies larger than $20 \mathrm{MeV}$, the optimal parameters cannot reproduce the nn phase shift in the low energy region $\left(e_{c . m} .<2 \mathrm{MeV}\right)$ and in the higher energy region simultaneously. We mainly focus on the adjustment of the nn phase shift in the low energy region. At higher energies, or equivalently at higher densities in nuclear matter, the medium effects modify the interaction anyhow and generate a density dependent term in the interaction.

Fixing $e_{c}$ and $a_{n n}$, one can deduce the effective range $r_{n n}=4 / \pi k_{c}$, the parameter $\alpha$ and the interaction strength $v_{0}=2 \pi^{2} \alpha \hbar^{2} / m$. The values of those parameters are given in Table [1. The value of the free interaction parameter $v_{0}^{*}$ deduced from the empirical value of the scattering length $a_{n n}=-18.5 \mathrm{fm}$ is also indicated. One can see that the difference between $v_{0}$ and $v_{0}^{*}$ is small, as much as $3 \%$. Indeed, as we are in a regime of large scattering length, one can deduce the interaction strength approximately from the relation $v_{0} \approx$ $v_{0}^{\infty}\left(1+\pi / 2 k_{c} a_{n n}+\ldots\right)$ where $v_{0}^{\infty}=-2 \pi^{2} \hbar^{2} / m k_{c}$ is the interacting strength in the unitary limit $\left(k_{c} a_{n n} \rightarrow \infty\right)$.

\section{B. The density-dependent function $g\left[\rho_{n}, \rho_{p}\right]$}

The density-dependent function $g$ is adjusted to reproduce the pairing gaps in symmetric and neutron matter 
obtained from Ref. [11]. Pairing in uniform nuclear matter is evaluated with the BCS ansatz:

$$
|B C S\rangle=\prod_{k>0}\left(u_{k}+v_{k} \hat{a}_{k \uparrow}^{\dagger} \hat{a}_{-k \downarrow}^{\dagger}\right)|-\rangle
$$

where $u_{k}$ and $v_{k}$ represent the BCS variational parameters and $\hat{a}_{k \uparrow}^{\dagger}$ are creation operators of a particle with momentum $k$ and spin $\uparrow$ on top of the vacuum $|-\rangle$ [35, 36, 37]. The BCS equations are deduced from the minimization of the energy with respect to the variational parameters $u_{k}$ and $v_{k}$. For a contact interaction, the equation for the pairing gap $\Delta_{n}$ takes the following simple form at zero temperature,

$$
\Delta_{n}=-\frac{v_{0} g\left[\rho_{n}, \rho_{p}\right]}{2(2 \pi)^{3}} \int d^{3} k \frac{\Delta_{n}}{E_{n}(k)} \theta(k, k),
$$

where $\theta(k, k)$ is the cutoff function associated to the contact interaction (2), $E_{n}(k)=\sqrt{\left(\epsilon_{n}(k)-\nu_{n}\right)^{2}+\Delta_{n}^{2}}$ is the neutron quasi-particle energy, $\epsilon_{n}(k)=\hbar^{2} k^{2} / 2 m_{n}^{*}$ is the neutron single particle kinetic energy with the effective mass $m_{n}^{*}$. We define the effective neutron chemical potential $\nu_{n}=\mu_{n}-U_{n}$, where the neutron mean field potential $U_{n}$ is subtracted from the neutron chemical potential $\mu_{n}$. The effective neutron chemical potential $\nu_{n}$ gives the neutron density,

$$
\rho_{n}=\frac{2}{V} \sum_{k} n_{n}(k)
$$

where $V$ is the volume and $n_{n}(k)$ is the occupation probability defined as

$$
n_{n}(k)=\frac{1}{2}\left[1-\frac{\epsilon_{n}(k)-\nu_{n}}{E_{n}(k)}\right] .
$$

Finally, the neutron Fermi momentum $k_{F n}$ is defined as $\rho_{n} \equiv k_{F n}^{3} / 3 \pi^{2}$.

We have chosen to adjust our interaction to the results of nuclear matter pairing gaps in Ref. [11] since it is the only calculations performed for both symmetric and neutron matter. We adjust the contact pairing interaction so that it reproduces the position and the absolute values of the maxima of the pairing gaps in symmetric and neutron matter. For the bare pairing gap, the maximum is located at $k_{F n}=0.87 \mathrm{fm}^{-1}$ with $\Delta_{n}=3.1 \mathrm{MeV}$ for both symmetric and neutron matter, while for the screened pairing gap, the maximum is at $k_{F n}=0.60 \mathrm{fm}^{-1}$ with $\Delta_{n}=2.70 \mathrm{MeV}$ for symmetric matter and $k_{F n}=0.83 \mathrm{fm}^{-1}$ and $\Delta_{n}=1.76 \mathrm{MeV}$ for neutron matter. The value of the parameters $\eta_{s}$ and $\eta_{n}$ are freely explored in the real axis while the parameters $\alpha_{s}$ and $\alpha_{n}$ are imposed to be positive to avoid singularities. The neutron effective mass $m_{n}^{*}$ is obtained from SLy4 Skyrme interaction since it is widely used in nuclear mean-field calculations. The results of the fits are given in Table I and the pairing gaps are shown in Fig. 2,

One should note that for the bare interaction, even if the pairing gap is identical in symmetric and neutron

\begin{tabular}{cccccc}
\hline \hline & $E_{c}=e_{c} / 2$ & $\eta_{s}$ & $\alpha_{s}$ & $\eta_{n}$ & $\alpha_{n}$ \\
\hline bare & $60 \mathrm{MeV}$ & 0.598 & 0.551 & 0.947 & 0.554 \\
$g=g_{1}$ & $40 \mathrm{MeV}$ & 0.664 & 0.522 & 1.01 & 0.525 \\
& $20 \mathrm{MeV}$ & 0.755 & 0.480 & 1.10 & 0.485 \\
& $10 \mathrm{MeV}$ & 0.677 & 0.365 & 0.931 & 0.378 \\
\hline screened-I & $60 \mathrm{MeV}$ & 7.84 & 1.75 & 0.89 & 0.380 \\
$g=g_{1}$ & $40 \mathrm{MeV}$ & 8.09 & 1.69 & 0.94 & 0.350 \\
& $20 \mathrm{MeV}$ & 9.74 & 1.68 & 1.00 & 0.312 \\
& $10 \mathrm{MeV}$ & 14.6 & 1.80 & 0.92 & 0.230 \\
\hline screened-II & $60 \mathrm{MeV}$ & 1.61 & 0.23 & 1.56 & 0.125 \\
$g=g_{1}+g_{2}$ & $40 \mathrm{MeV}$ & 1.80 & 0.27 & 1.61 & 0.122 \\
$\eta_{2}=0.8$ & $20 \mathrm{MeV}$ & 2.06 & 0.31 & 1.70 & 0.122 \\
& $10 \mathrm{MeV}$ & 2.44 & 0.37 & 1.66 & 0.0939 \\
\hline \hline
\end{tabular}

TABLE II: Parameters of the function $g$ defined in Eq. (2). These parameters are obtained from the fit to the pairing gaps in symmetric and neutron matter. These are the parameters obtained from the adjustment of the bare gap and the screened gap with $g=g_{1}$, and the screened gap including the additional function $g_{2}$. The effective mass is obtained from SLy4 Skyrme interaction. Note that $E_{c}$ is the cutoff for the quasi-particle gap equation (6) while $e_{c}$ is that for the twobody scattering so that $E_{c}=e_{c} / 2$. See the text for details.

matter, the adjusted contact interaction is not necessarily isoscalar. Indeed, the transformation from the Fermi momentum, the $\mathrm{x}$-axis of Fig. 2, to the density is different in symmetric nuclear matter, $\rho / \rho_{0}=\left(k_{F n} / k_{F 0}\right)^{3}$ (where $\left.\rho_{0}=2 /\left(3 \pi^{2}\right) k_{F 0}^{3}=0.16 \mathrm{fm}^{-3}\right)$, and in neutron matter, $\rho / \rho_{0}=0.5\left(k_{F n} / k_{F 0}\right)^{3}$. Therefore, an interaction which depends only on the ratio $\rho / \rho_{0}$ gives different results if it is plotted as a function of $k_{F n}$ in symmetric and neutron matter. As the pairing gap calculated with the bare interaction [11] is quasi-identical in symmetric and neutron matter when it is plotted versus $k_{F n}$, one can then deduce the following relations between the parameters of the density-dependent term $g_{1}$ (neglecting the isospin dependence of the effective mass): $\alpha_{s}=\alpha_{n}$ and $\eta_{s}=\eta_{n} / 2^{\alpha_{n}}$.

For the bare pairing gap and for a given cutoff energy $E_{c}$, the position and the maximum value of the gap are reproduced well by the contact interaction in Fig. 2, However, in the high Fermi momentum region $k_{F n}>1 \mathrm{fm}^{-1}$, we can see appreciable difference between the microscopic predictions and the pairing gap obtained from the contact interactions. The best agreement is obtained for a cutoff energy $E_{c}=40 \mathrm{MeV}$. In the screened case, the dependence of the pairing gap on $k_{F n}$ is badly reproduced, especially for symmetric nuclear matter. This is because the maximum position of the pairing gap is shifted towards a lower neutron Fermi momentum (one third in density from that for the bare gap). Consequently, the density dependence of the function $g_{1}$ becomes stiffer in the "screened" case than in the bare case, and the gap 


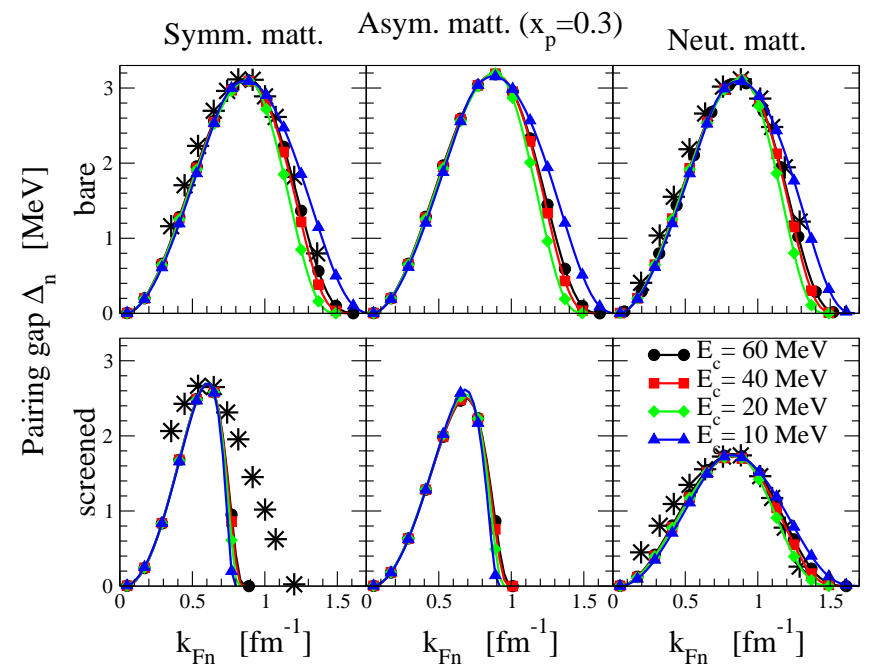

FIG. 2: (Color online) Pairing gap in symmetric, asymmetric $\left(x_{p}=\rho_{p} / \rho\right)$ and neutron matter adjusted to the "bare gap" (upper panel) or to the "screened gap" (lower panel) for various cutoff energies $E_{c}$. The pairing gap calculated from the microscopic treatment presented in Ref. [1] is also shown as star symbols.

drops faster after the maximum, as it is shown in Fig. 2 This may indicate that the screened interaction has a different density dependence and cannot be cast into a simple power law of the density as in Eq. (2). Indeed, in Ref. [11], the medium polarization effects have been analyzed at the level of the interacting potential, and it was shown that the medium polarization effects emerge at very low density and remain relatively constant. To simulate such effects, it seems necessary to introduce a new term, $g_{2}$ in Eq. (2). We propose for $g_{2}$ a simple isoscalar constant which switches on at a very low value of the density $\left(k_{F} \sim 0.15 \mathrm{fm}^{-1}\right)$ and switch off around the saturation density. The following form satisfies this condition,

$$
g_{2}=\eta_{2}\left[\left(1+e^{\frac{k_{F}-1.4}{0.05}}\right)^{-1}-\left(1+e^{\frac{k_{F}-0.1}{0.05}}\right)^{-1}\right] .
$$

The new pairing interaction with $g=g_{1}+g_{2}$, hereafter named screened-II, has then only one new adjustable parameter $\eta_{2}$. As the medium polarization effects could also change the density-dependent term $g_{1}$, all the 5 parameters have to be re-adjusted. In Tables II and III. we give the new parameters $\eta_{s}, \alpha_{s}, \eta_{n}$ and $\alpha_{n}$, obtained for several values of $\eta_{2}$. The cutoff energy is fixed to be $E_{c}=40 \mathrm{MeV}$. The corresponding pairing gap is represented in Fig. 3. The best fit is obtained for the value $\eta_{2}=0.8$. Eq. (9) may not be a unique way to take into account the medium polarization effects. Nevertheless it is simple enough to apply to the BCS-BEC crossover, so this is why we adopt this functional form.

Solving the gap Eq. (6), the neutron effective chemical potential $\nu_{n}$ is determined for a given interaction at

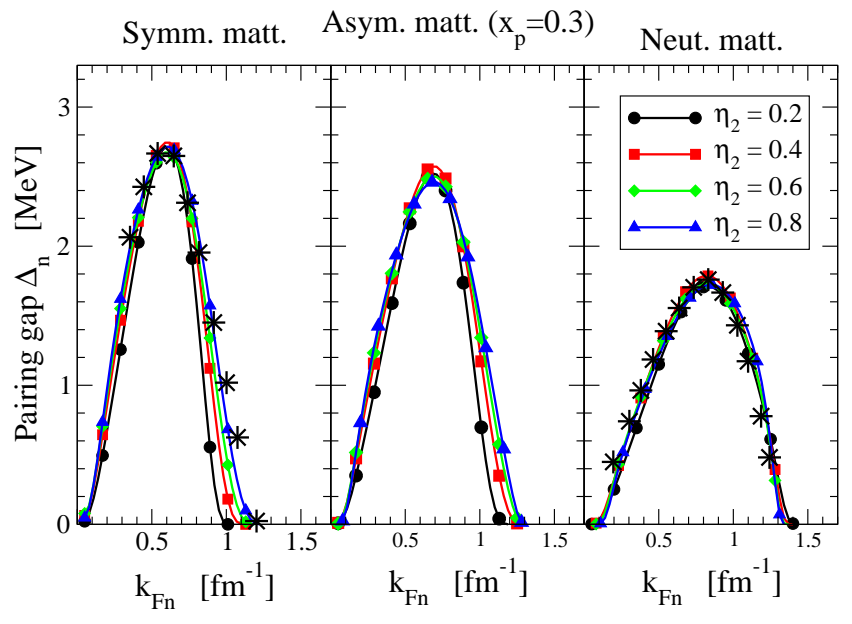

FIG. 3: (Color online) Pairing gap calculated in symmetric, asymmetric and neutron matter with the screened-II interaction $\left(g=g_{1}+g_{2}\right)$ and for several values of $\eta_{2}$ as indicated in the legend. The corresponding parameters are given in Table III See the text for details.

a given neutron Fermi momentum $k_{F n}$. The neutron effective mass $m_{n}^{*}$, the effective neutron chemical potential $\nu_{n}=\mu_{n}-U_{n}$ and the difference $\nu_{n}-\epsilon_{F n}$ are represented in Fig. 4 as a function of the neutron Fermi momentum in symmetric and neutron matter. The neutron effective mass and the neutron potential $U_{n}$ are deduced from the SLy4 Skyrme interaction. Note that the neutron density $\rho_{n}$ is changed into the neutron Fermi momentum, $k_{F n}$. We have selected from Table II the bare and the screenedII pairing interactions for a cutoff energy $E_{c}=40 \mathrm{MeV}$. In the absence of pairing, the effective neutron chemical potential $\nu_{n}$ is identical to the neutron Fermi kinetic energy, $\nu_{n}=\epsilon_{F n}$ where $\epsilon_{F n}=\epsilon_{n}\left(k_{F n}\right)$. The difference $\nu_{n}-\epsilon_{F n}$ is then null in the absence of pairing correlations, otherwise it is negative as shown in Fig. 4. From this difference, one can estimate the relative importance of the pairing correlations: in neutron matter the screened-II interaction leads to weaker pairing correlations compared

\begin{tabular}{ccccc}
\hline \hline$\eta_{2}$ & $\eta_{s}$ & $\alpha_{s}$ & $\eta_{n}$ & $\alpha_{n}$ \\
\hline 0.2 & 1.90 & 0.72 & 1.08 & 0.24 \\
0.4 & 1.61 & 0.46 & 1.26 & 0.19 \\
0.6 & 1.64 & 0.33 & 1.44 & 0.15 \\
0.8 & 1.80 & 0.27 & 1.61 & 0.122 \\
\hline \hline
\end{tabular}

TABLE III: Parameters of the the screened-II interaction, where the density-dependent function of the pairing interaction is taken to be $g=g_{1}+g_{2}$. The functional forms $g_{1}$ and $g_{2}$ are obtained by fitting the screened pairing gap for several values of $\eta_{2}$. The energy cutoff is taken to be $E_{c}=40 \mathrm{MeV}$ and the neutron effective mass is deduced from the SLy4 Skyrme interaction. 

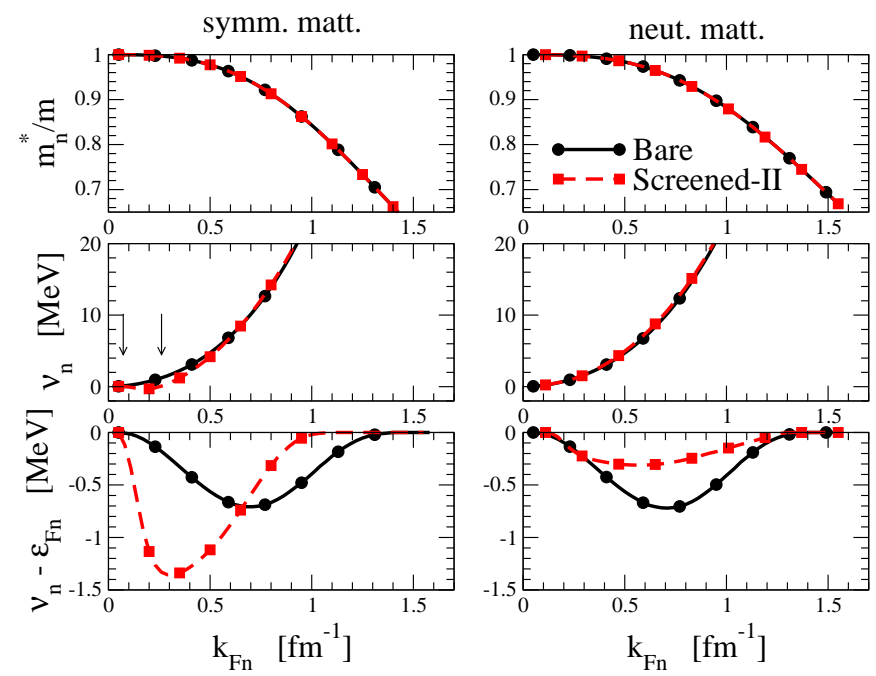

FIG. 4: (Color online) Comparison of the neutron effective mass $m_{n}^{*}$, the effective chemical potential $\nu_{n}=\mu_{n}-U_{n}$, and the difference $\nu_{n}-\epsilon_{F n}$ calculated with the bare and the screened-II contact interactions. The parameters of the pairing interactions are taken from Table II with the cutoff energy $E_{c}=40 \mathrm{MeV}$. The effective mass $m_{n}^{*}$ and the neutron potential $U_{n}$ are taken from SLy4 Skyrme interaction. The two arrows indicate the lower and upper limits for the condition $\nu_{n}<0$ with the screened-II interaction in symmetric nuclear matter.

to the bare one, while in symmetric matter, the screenedII interaction give much stronger pairing correlations for $k_{F n}<0.7 \mathrm{fm}^{-1}$ and less for $k_{F n}>0.7 \mathrm{fm}^{-1}$.

It is easy to show that the gap (6) and the occupation probability (8) go over into the Schrödinger-like equation for the neutron pair wave function $\Psi_{\text {pair }}$ [38],

$$
\frac{p^{2}}{m} \Psi_{\text {pair }}+\left[1-2 n_{n}(k)\right] \frac{1}{V} \operatorname{Tr} v_{n n} \Psi_{\text {pair }}=2 \nu_{n} \Psi_{\text {pair }}
$$

See Eq. (13) in Sec. III for a proper definition of the neutron pair wave function $\Psi_{\text {pair }}$. Notice that, at zero density where $n_{n}(k)=0$, Eq. (10) is nothing but the Schrödinger equation for the neutron pair. From Eq. (10), one usually relates the effective neutron chemical potential $\nu_{n}$ to be a half of the "binding energy" of a Cooper pair. The Cooper pairs may then be considered to be strongly correlated if $\nu_{n}$ is negative. Notice from Fig. 4, that the effective neutron chemical potential $\nu_{n}$ becomes negative with the screened-II interaction in symmetric nuclear matter for $k_{F n}=0.05-0.35 \mathrm{fm}^{-1}$, but not at all in neutron matter. One could then expect in this case that the Cooper pair may resemble a closely bound system (BEC) in symmetric nuclear matter at very low density while in neutron matter it should behave like that of the weak coupling BCS regime. However, to go beyond this rough interpretation, we need to study more accurately the BCS-BEC crossover in asymmetric matter.

\begin{tabular}{cccccc}
\hline \hline$\left(k_{F n} a_{n n}\right)^{-1}$ & $P\left(d_{n}\right)$ & $\xi_{r m s} / d_{n}$ & $\Delta_{n} / \epsilon_{F n}$ & $\nu_{n} / \epsilon_{F n}$ & \\
\hline-1 & 0.81 & 1.10 & 0.21 & 0.97 & BCS boundary \\
0 & 0.99 & 0.36 & 0.69 & 0.60 & unitarity limit \\
1 & 1.00 & 0.19 & 1.33 & -0.77 & BEC boundary \\
\hline \hline
\end{tabular}

TABLE IV: Reference values of $\left(k_{F n} a_{n n}\right)^{-1}, P\left(d_{n}\right), \xi_{r m s} / d_{n}$, $\Delta_{n} / \epsilon_{F n}$ and $\nu_{n} / \epsilon_{F n}$ characterizing the BCS-BEC crossover in the regularized model for the contact interaction. The values $d_{n}, P\left(d_{n}\right)$, and $\xi_{\text {rms }}$ are the average distance between neutrons $d_{n}=\rho_{n}^{-1 / 3}$, the probability for the partner neutrons correlated within the relative distance $d_{n}$, and the rms radius of Cooper pair, respectively. The numbers have been taken from Refs. 25, 40]. See the text for details.

\section{APPLICATION TO THE BCS-BEC CROSSOVER}

Going from the weak coupling BCS regime, around the saturation density $\rho_{0}$, down to the BCS-BEC crossover, for densities $<\rho_{0} / 10$, it has been shown that the spatial structure of the neutron Cooper pair changes [25]. It is indeed expected that the correlations between two neutrons get large as the density decreases and as a consequence, the BCS-BEC crossover occurs in the uniform matter at low density. However, being of the second order, this transition is smooth. Hereafter, we clarify the boundaries of the BCS-BEC phase transition by using a regularized gap equation.

Although the BCS ansatz (5) has been developed to describe the Cooper pair formation in the weak BCS regime [35], it has been shown that the BCS equations are also valid in the strong BEC condensation regime [38, 39]. The BCS equations are thus adopted as a useful framework to describe the intermediate BCS-BEC crossover regime at zero temperature [40]. It has been proposed to define the limit of the BCS-BEC phase transition using a regularized model for the pairing gap [25, 40, 41]. In this model, the BCS gap (6) is combined with the relation between the interaction strength and the scattering length which has a similar divergent behavior. The difference between those two divergent integrals gives a regularized equation,

$$
\frac{m_{n}}{4 \pi a_{n n}}=-\frac{1}{2 V} \operatorname{Tr}\left(\frac{1}{E_{n}(k)}-\frac{1}{\epsilon_{n}(k)}\right),
$$

which has no divergence. The gap equation (6) can be solved analytically for the contact interaction with a constraint of the particle number conservation (77). The solution of this regularized gap equation is independent of the strength of the interaction, and the gap is uniquely determined by the value of the scattering length $a_{n n}$. From Eq. (11), one can study the boundaries of the BCS-BEC phase transition with respect to the dimensionless order parameter $k_{F n} a_{n n}$. We give in Table IV the values of several quantities which specify the phase transition: the 
probability $P\left(d_{n}\right)$ for the partner neutrons to be correlated within the relative distance $d_{n}\left(d_{n}\right.$ is the average distance between neutrons $d_{n}=\rho_{n}^{-1 / 3}$ ), the ratio of the rms radius to the mean neutron distance $\xi_{r m s} / d_{n}$, the ratio of the pairing gap to the single particle kinetic energy $\Delta_{n} / \epsilon_{F n}$ and also the ratio of the effective neutron chemical potential to the single particle kinetic energy $\nu_{n} / \epsilon_{F n}$. As we already mentioned, these boundaries are indicative because the phase transition is smooth at the boundaries being of the second order. For instance, even if the nuclear matter does not enter into the BEC regime, we will show that the Cooper pair wave function is already very similar to the BEC one when it is close.

A drawback of this regularized model is that the relation between the dimensionless order parameter $k_{F n} a_{n n}$ and the density of the medium is unknown. To relate the order parameter to the density, one has to re-introduce the pairing strength in the gap equation (6). We could consider for instance a contact interaction with a cutoff regularization. The density will then trigger the phase transition for a given pairing interaction strength. In the following, we study the BCS-BEC phase diagram in asymmetric nuclear matter for the two pairing interactions discussed in Sec. [II Namely we explore the properties of the Cooper pair wave function obtained by the bare and the screened-II interactions presented in Table $\amalg$ for a fixed cutoff energy $E_{c}=40 \mathrm{MeV}$.

The BCS approximation provides the Cooper pair wave function $\Psi_{\text {pair }}(k)$ [35, 36, 37]

$$
\begin{aligned}
\Psi_{\text {pair }}(k) & \equiv C\left\langle B C S\left|\hat{a}^{\dagger}(k \uparrow) \hat{a}^{\dagger}(-k \downarrow)\right| B C S\right\rangle \\
& \equiv C u_{k} v_{k} .
\end{aligned}
$$

The radial shape of the Cooper pair wave function is deduced from the Fourier transform of $u_{k} v_{k}=\Delta_{n} / 2 E_{n}(k)$ in Eq. (13). The rms radius of Cooper pairs is then given by $\xi_{r m s}=\sqrt{\left\langle r^{2}\right\rangle}=\sqrt{\int d r r^{4}\left|\Psi_{\text {pair }}(r)\right|^{2}}$. The rms radius $\xi_{r m s}$ and the Pippard's coherence length, $\xi_{P}=\hbar^{2} k_{F n} / m_{n}^{*} \pi \Delta_{n}$, give similar size of the Cooper pair in the weak coupling regime. The rms radius $\xi_{r m s}$ is nevertheless a more appropriate quantity in the BCS-BEC crossover region as well as in the strong BEC coupling region. In order to estimate the size of Cooper pairs a reference scale is given by the average distance between neutrons $d_{n}$. If the rms radius of Cooper pairs is larger than $d_{n}$, the pair is interpreted as an extended BCS pair while the Cooper pair will be considered as a compact BEC pair if the rms radius is smaller than the average distance. Let us introduce another important quantity which also gives a measure of the spatial correlations: the probability $P(r)$ for the partners of the neutron Cooper pair to come close to each other within the relative distance $r$,

$$
P(r)=\int_{0}^{r} d r^{\prime} r^{2}\left|\Psi_{\text {pair }}\left(r^{\prime}\right)\right|^{2} .
$$

The order parameters listed in Table IV are closely related. For instance, approximating $\xi_{r m s}$ by $\xi_{P}$, it could easily be shown that the ratio $\xi_{r m s} / d_{n}$ is proportional to $\epsilon_{F n} / \Delta_{n}$. Then, the strong coupling regime is reached if the ratio $\Delta_{n} / \epsilon_{F n}$ is large. The order parameter $\nu_{n}$, the effective neutron chemical potential, could be interpreted as a half of the binding energy of Cooper pairs at finite density according to the Schrödinger-like Eq. (10).

As shown in the Appendix Bit is convenient to decompose the Cooper pair wave function into

$$
\Psi_{\text {pair }}(r)=\Psi_{1}(r)+\Psi_{2}(r),
$$

where

$$
\begin{aligned}
& \Psi_{1}(r)=C^{\prime} \int_{0}^{k_{\infty}} d k \frac{k^{2}}{E_{n}(k)} \frac{\sin (k r)}{k r}, \\
& \Psi_{2}(r)=C^{\prime} \int_{k_{\infty}}^{\infty} d k \frac{k^{2}}{E_{n}(k)} \frac{\sin (k r)}{k r} .
\end{aligned}
$$

Choosing $k_{\infty} / k_{0} \gg 1$, with $k_{0}=\sqrt{2 m_{n}^{*} \nu_{n}} / \hbar$, it is possible to find an analytic expression for $\Psi_{2}$ :

$$
\Psi_{2}(r)=-\frac{C^{\prime}}{r} \frac{2 m_{n}^{*}}{\hbar^{2}} \operatorname{si}\left(k_{\infty} r\right),
$$

where $\operatorname{si}(u)$ is the sinus integral defined as $\operatorname{si}(u)=$ $\int_{u}^{\infty} d z[\sin (z) / z]$. It is clear from Eq. (18) that the term $\Psi_{2}$ has a $1 / r$-type singularity. This singularity is due to the nature of the contact interaction which does not contain a hard core repulsion. With the hard core repulsion, the wave function goes to zero as $r \rightarrow 0$ [25]. In the outer region $(r>3 \mathrm{fm})$, the wave function behaves in the same way if the contact interaction is deduced properly from the microscopic calculations. We checked the convergence of the wave function (15) with respect to the parameter $k_{\infty}$. We found that the convergence is reached with $k_{\infty} \approx 2 k_{0}$ as is is shown in Fig. [1] in the Appendix B. In Ref. [25], Matsuo introduced the cutoff momentum $k_{c}$ to calculate the pair wave function (15). We have compared the pair wave function $\Psi_{\text {pair }}(r)$ with the one obtained by Matsuo. The two wave functions give essentially the same results, except for the low density region. In the worst case, the wave function of Matsuo's treatment increases the rms radius by about $10 \%$ compared to the one obtained by the wave function (15).

The neutron Cooper pair wave function $r^{2}\left|\Psi_{\text {pair }}(r)\right|^{2}$ is shown in Fig. 5 as a function of the relative distance $r$ between the pair partners taking different Fermi momenta $k_{F n}=1.1,0.8,0.5$ and $0.2 \mathrm{fm}^{-1}$, which correspond respectively to the densities: $\rho_{n} / \rho_{0}=0.3,0.1,0.03$ and 0.002. Calculations in symmetric, asymmetric and neutron matter are shown in the left, middle and right panels, respectively. In Fig. 5 , we observe that the spatial extension and the profile of the Cooper pair varies strongly with the density. A large extension is found close to the saturation density at $k_{F n}=1.1 \mathrm{fm}^{-1}$. The profile of the wave function behaves as an oscillation convoluted by a decreasing exponent and casts into the well known limit $\sim K_{0}\left(r / \pi \xi_{P}\right) \sin \left(k_{F} r\right) / k_{F} r$ 35. This indicates that the Cooper pair is in the weak coupling BCS regime. At lower 
symm. matt. asymm. matt. $\left(\mathrm{x}_{\mathrm{p}}=0.3\right)$

neut. matt.

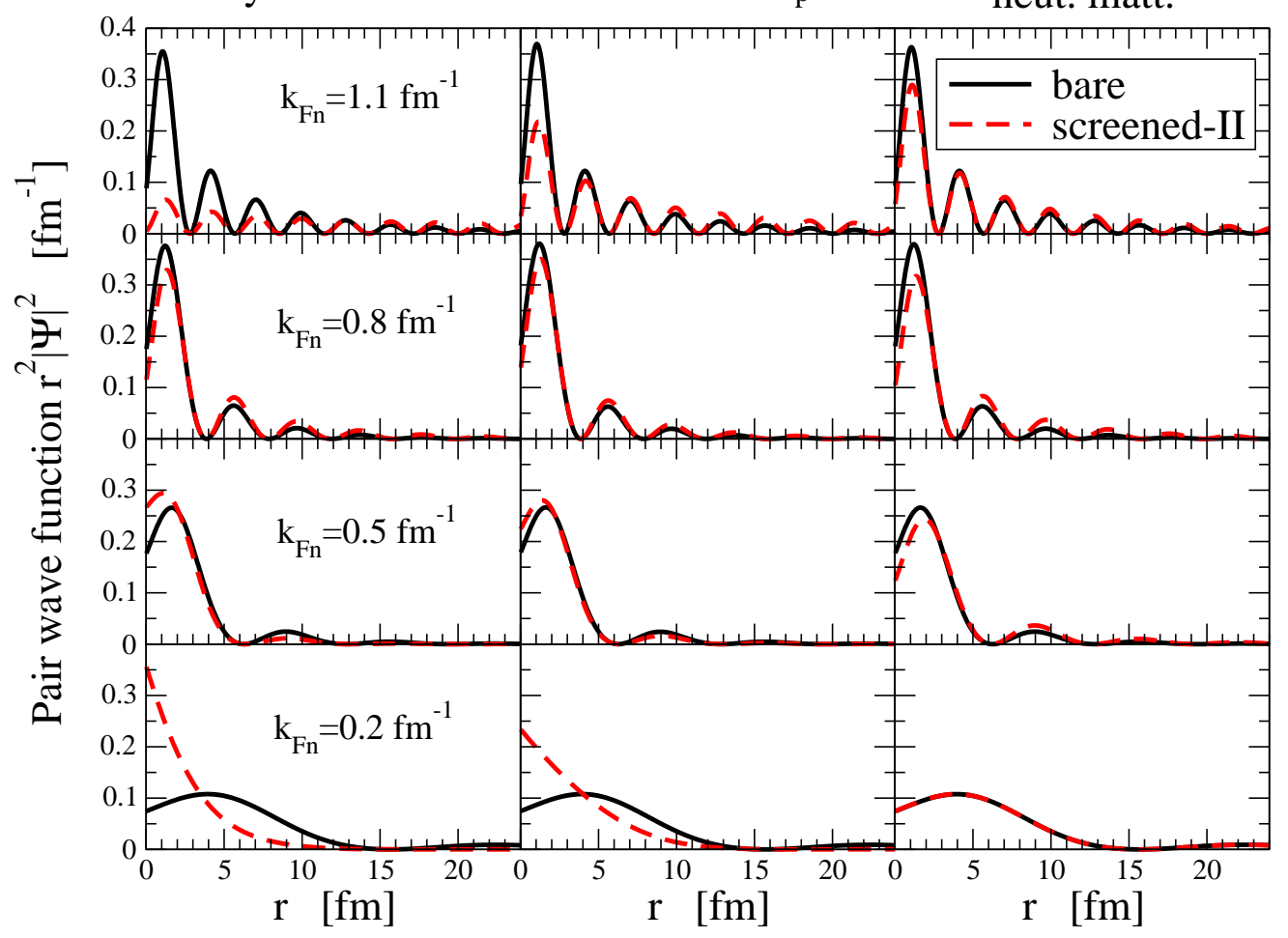

FIG. 5: (Color online) Neutron Cooper pair wave function $r^{2}\left|\Psi_{\text {pair }}(r)\right|^{2}$ as a function of the relative distance $r$ between the pair partner at the Fermi momenta $k_{F n}=1.1,0.8,0.5$ and $0.2 \mathrm{fm}^{-1}$.

densities, the Cooper pair shrinks and the oscillation disappears. The wave function resembles now the strong coupling limit $(\mathrm{BEC}) \sim \exp \left(-\sqrt{4 m / \hbar^{2}|\mu|} r\right) / r$ [39]. This is an indication that a possible BCS-BEC crossover may occur in uniform matter.

It should be remarked that the latter limit seems well pronounced in symmetric matter with the screened gap (see the panel at the left bottom corner of Fig. (5). We show in Fig. [6] the evolution of the occupation probability (8) in symmetric matter for the two pairing interactions. For the screened-II interaction, the pairing correlations becomes strong at low densities as the occupation probability is considerably different from the step function. In the case of the bare interaction, the correlations are not so strong to change $n_{n}(k)$ drastically even at low densities. It should be noticed that this analysis is independent of the detailed structure of the Cooper pair wave function. This change of the occupation probability proves that the behavior of the Cooper pair wave function is not an artifact induced by the zero range behavior of the contact interaction but indeed is physical. It is clear that low density symmetric nuclear matter is much more correlated with the screened-II interaction than with the bare one. This is also the case for the BCS-BEC crossover as will be discussed below.

Let us now discuss the BCS-BEC crossover which may depend on the pairing interactions and also on the asym- metry of the nuclear medium. In the following, we study the different order parameters in Table IV for the boundaries of the BCS-BEC phase transition. Fig. 7 shows the
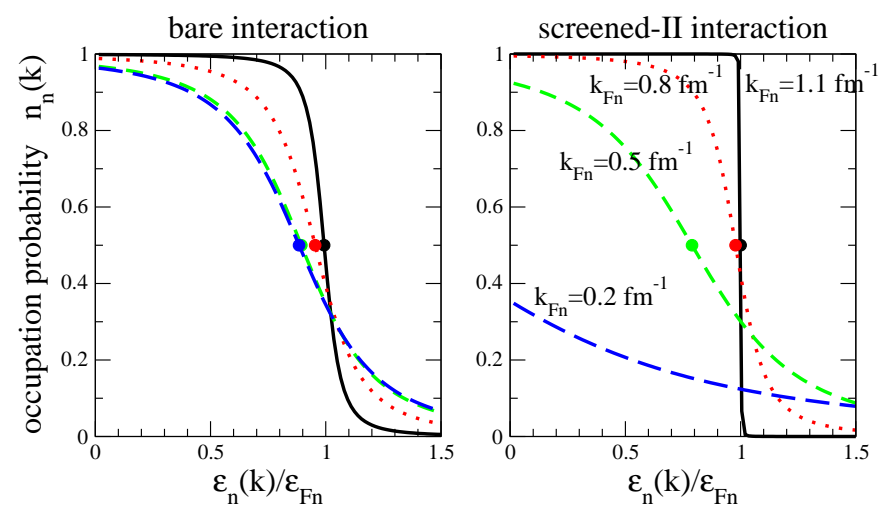

FIG. 6: (Color online) Occupation probability $n_{n}(k)$ for symmetric matter defined by Eq. (8) as a function of the ratio of the single particle kinetic energy to the Fermi energy $\epsilon_{n}(k) / \epsilon_{F n}$ for a set of Fermi momenta $k_{F n}=1.1,0.8,0.5$ and $0.2 \mathrm{fm}^{-1}$. We compare the results of the bare interaction (left panel) with the screened-II interaction (right panel). The values of the effective neutron chemical potential $\nu_{n} / \epsilon_{F n}$ are indicated by the filled circles. 


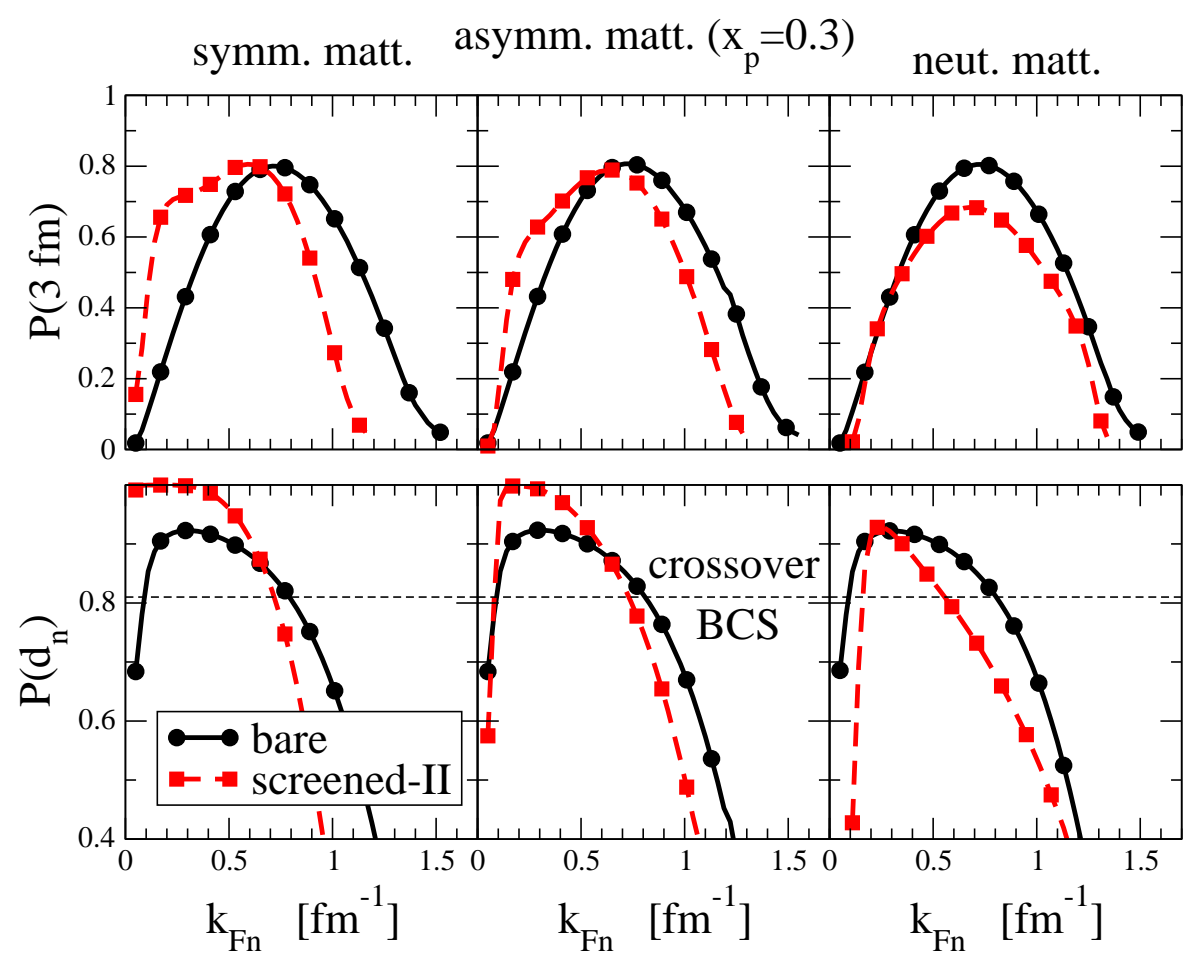

FIG. 7: (Color online) Probability $P$ for the partner neutrons within two typical lengths, 3 fm and $d_{n}$, as a function of the neutron Fermi momentum $k_{F n}$ in symmetric (left panel), asymmetric (central panel) and neutron matter (right panel). The boundary of the BCS-BEC crossover is denoted by the dashed line.

probabilities $P(r)$ for the partner neutrons to be correlated within the typical scales, $r=3 \mathrm{fm}$ and $r=d_{n}$. The former scale is the typical range of the nucleon-nucleon force. For the bare interaction, the probability $P(3 \mathrm{fm})$ has similar behavior in symmetric and asymmetric matter as a function of $k_{F n}$. For the screened-II interaction, there is a noticeable isospin dependence. A low density shoulder appears in symmetric matter, at around $k_{F n} \sim 0.25 \mathrm{fm}^{-1}\left(\rho_{n} / \rho_{0} \sim 0.003\right)$. Then, it becomes smaller as the asymmetry increases and eventually disappears in neutron matter. In neutron matter, the strong concentration of the pair wave function within the interaction range $3 \mathrm{fm}, P(3 \mathrm{fm})>0.5$, is realized in the density region $k_{F n} \sim 0.3-1.1 \mathrm{fm}^{-1}$ (or $\rho_{n} / \rho_{0} \sim 0.007-0.3$ ) for both pairing interactions. For symmetric matter, on the other hand, this region is different for the two pairing interactions: the strong correlation occurs at much lower density region for the screened-II interaction than for the bare one. This property can also be confirmed by the probability $P\left(d_{n}\right)$. For the two pairing interactions, the Cooper pairs in symmetric and asymmetric matter enter into the crossover regime at almost the same density. The crossover in neutron matter occurs somewhat at lower density for the screened-II interaction. As the density decreases, a different behavior is observed between the two pairing interactions for symmetric matter. While the probability $P\left(d_{n}\right)$ decreases and goes back to the weak BCS regime for the bare interaction at very small density below $k_{F n} \sim 0.1 \mathrm{fm}^{-1}$, the probability $P\left(d_{n}\right)$ continue to increase up to 1 for the screened-II interaction at very low densities, $k_{F n}<0.7 \mathrm{fm}^{-1}\left(\rho=n / \rho=0<0.07 \mathrm{fm}^{-1}\right)$.

We study further the BCS-BEC crossover by looking at the rms radius $\xi_{r m s}$ and the neutron pairing gap $\Delta_{n}$. In Fig. 8, we show the rms radius $\xi_{r m s}$ as a function of the neutron Fermi momentum $k_{F n}$ as well as the order parameter $\xi_{r m s} / d_{n}$. The rms radius of the Cooper pair is less than $5 \mathrm{fm}$ in the region $k_{F n} \sim(0.4-0.9) \mathrm{fm}^{-1}$ $\left(\rho_{n} / \rho_{0} \sim 0.01-0.15\right)$ in the three panels for the bare interaction. The screened-II interaction gives different effects in symmetric and asymmetric matter: it increases the rms radius for the neutron matter, while the rms radius stays small around $4 \mathrm{fm}$ even at very low density at $k_{F n} \sim 0.15 \mathrm{fm}^{-1}\left(\rho_{n} / \rho_{0} \sim 0.0007\right)$ in symmetric matter. In the lower panels is shown the ratio of the rms radius to the average distance between neutrons $d_{n}$. For the bare interaction, the size of the Cooper pair becomes smaller than $d_{n}$ for the Fermi momentum $k_{F n}<0.8 \mathrm{fm}^{-1}$ $\left(\rho_{n} / \rho_{0} \sim 0.1\right)$ in general. There are substantial differences for symmetric and asymmetric matter in the case of the screened-II interaction. The crossover region becomes smaller for the neutron matter, while the crossover region increases in the cases of asymmetric $\left(x_{p}=0.3\right)$ and symmetric matter. Especially, the correlations becomes strong in symmetric matter and the Cooper pair reaches almost the BEC boundary at $k_{F n} \sim 0.2 \mathrm{fm}^{-1}$ $\left(\rho_{n} / \rho_{0} \sim 0.002\right)$. Notice that the two neutrons system is 
symm. matt. asymm. matt. $\left(\mathrm{x}_{\mathrm{p}}=0.3\right)$ neut. matt.
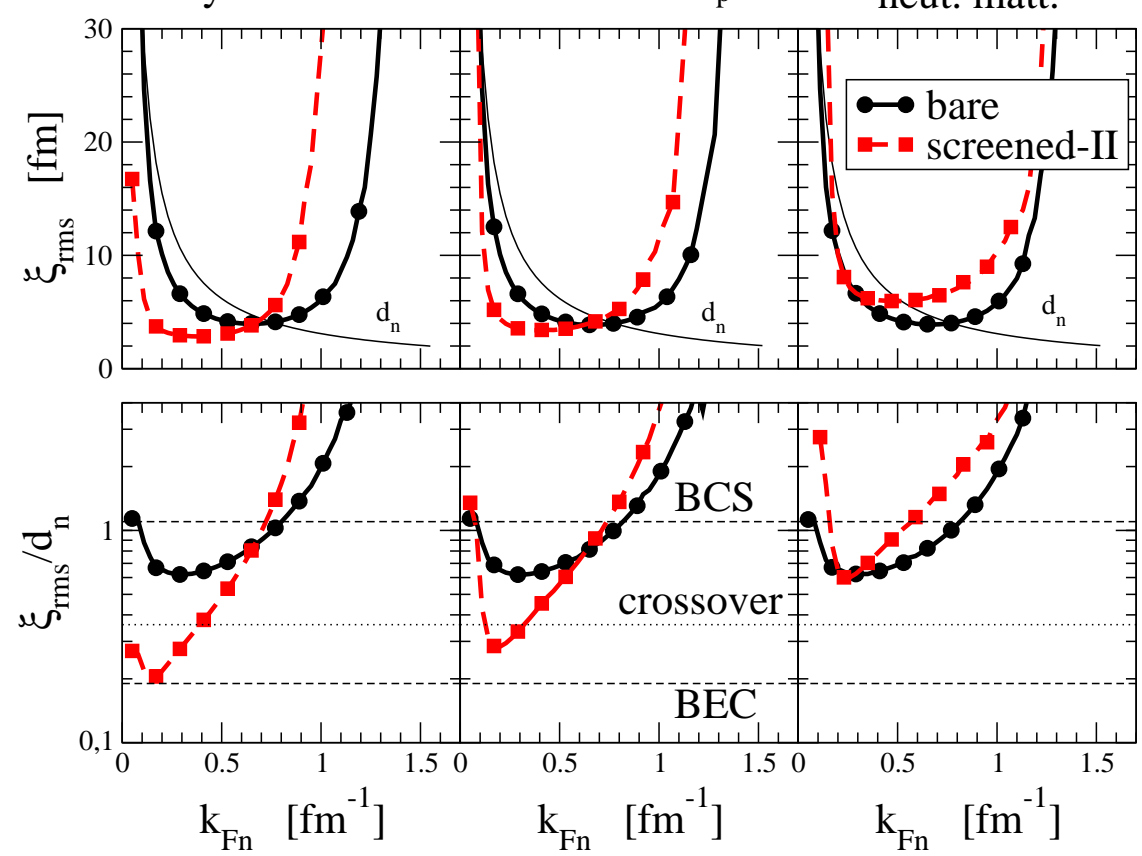

FIG. 8: (Color online) Top panels: Comparison between the rms radius $\xi_{r m s}$ of the neutron pair and the average inter-neutron distance $d_{n}=\rho_{n}^{-1 / 3}$ (thin line) as a function of the neutron Fermi momentum $k_{F n}$ in symmetric (left panel), asymmetric (central panel) and neutron matter (right panel). Bottom panels: The order parameter $\xi_{r m s} / d_{n}$ as a function of $k_{F n}$. The boundaries of the BCS-BEC crossover are represented by the two dashed lines, while the unitary limit is shown by the dotted line. The two pairing interactions are used for the calculations.
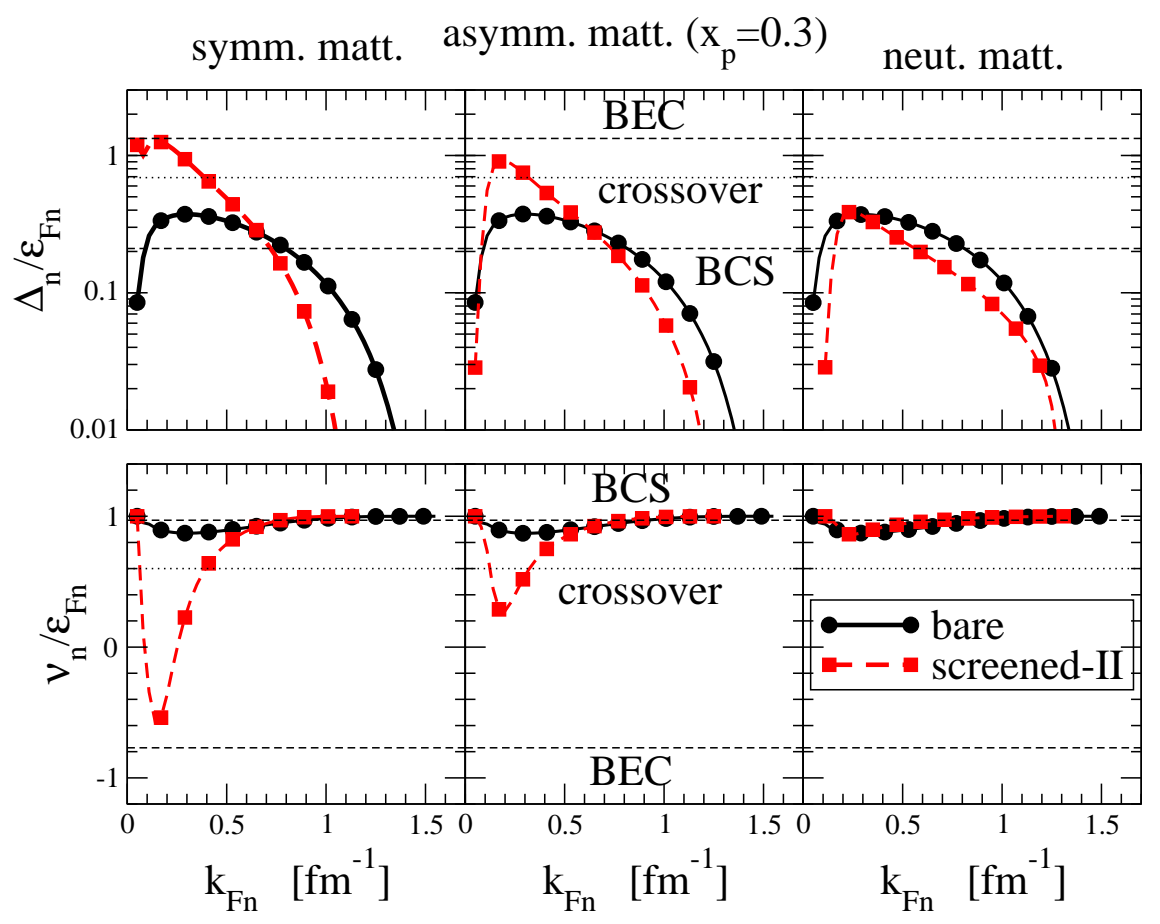

FIG. 9: (Color online) Ratios $\Delta_{n} / \epsilon_{F n}$ and $\nu_{n} / \epsilon_{F n}$ plotted as a function of the neutron Fermi momentum $k_{F n}$ in symmetric (left panel), asymmetric (central panel) and neutron matter (right panel). The boundaries of the BCS-BEC crossover are shown by the two dashed lines, while the unitary limit is given by the dotted line. See the text for details. 
known experimentally to have a virtual state in the zero density limit. We have shown that this virtual state could lead to a strongly correlated BEC state at low density in symmetric nuclear matter according to the screened-II interaction.

The two other order parameters $\Delta_{n} / \epsilon_{F n}$ and $\nu_{n} / \epsilon_{F n}$ are shown in Fig. 9, These results confirm the BCS-BEC crossover behavior which is found in Fig. 8. Namely, in symmetric matter, the gap $\Delta_{n}$ is much enhanced by the screened interaction in the low density region, while no enhancement can be seen in neutron matter. As expected, the effective chemical potential $\nu_{n}$ induced by the screened-II interaction becomes negative for $k_{F n} \sim$ $0.05-0.3 \mathrm{fm}^{-1}\left(\rho_{n} / \rho_{0} \sim 0.00002-0.01\right)$ in symmetric nuclear matter. This strong correlation is reduced in asymmetric matter and is almost absent in the neutron matter as can be seen in Fig. 9. It should also be remarked that the order parameter $\nu_{n} / \epsilon_{F n}$, due to the Schrödinger-like Eq. (10), gives the same BCS-BEC crossover behavior as that of the order parameter $\xi_{r m s} / d_{n}$. The neutron effective chemical potential is then a good criteria to discuss the BCS-BEC crossover.

\section{CONCLUSIONS}

A new type of density-dependent contact pairing interaction was obtained to reproduce the pairing gaps in symmetric and neutron matter obtained from a microscopic calculation [11]. The contact interactions reproduce the two types of pairing gaps, i.e., the gap calculated with the bare interaction and the gap modified by medium polarization effects. It is shown that the medium polarization effects cannot be cast into the usual density power law form in symmetric nuclear matter so that another new isoscalar term $g_{2}$ in Eq. (9) is then added to the density dependent term of the pairing interaction in Eq. (1).

We have applied these density-dependent pairing interactions to the study of the BCS-BEC crossover phenomenon in symmetric and asymmetric nuclear matter. We found that the spatial di-neutron correlation is strong in general in a wide range of low matter densities, up to $k_{F n} \sim 0.9 \mathrm{fm}^{-1}\left(\rho_{n} / \rho_{0} \sim 0.15\right)$. This result is independent of the pairing interaction, either bare or screened one, as well as of the asymmetry of the uniform matter. Moreover, it is shown that the two pairing interactions mentioned above lead to different features for BCS-BEC phase transition in symmetric nuclear matter. To clarify the difference, we studied various order parameters, the correlation probability $P\left(d_{n}\right)$, the rms radius of the Cooper pair $\xi_{r m s}$, the gap $\Delta_{n}$ and the effective chemical potential $\nu_{n}$, as a function of the Fermi momentum $k_{F n}$, or equivalently as a function of the density. The screened interaction enhances the BCS-BEC crossover phenomena in symmetric matter, while the pairing correlations as well as the crossover phenomena are decreased in neutron matter by the medium polarization effects. For the screened-II interaction, the crossover reaches almost to the BEC phase at $k_{F n} \sim 0.2 \mathrm{fm}^{-1}$ in symmetric matter. We should notice, however, that the BEC state is very sensible to the asymmetry of the medium and disappears in neutron matter.

\section{Acknowledgments}

We are grateful to M. Matsuo, P. Schuck and N. Sandulescu for interesting discussions during the completion of this work. This work was supported by the Japanese Ministry of Education, Culture, Sports, Science and Technology by Grant-in-Aid for Scientific Research under the program number 19740115.

\section{APPENDIX A: EFFECTS OF THE CUTOFF PRESCRIPTION ON PAIRING GAP}

In the gap (6), the integral runs over the momentum $k$, which is limited by the cutoff momenta $k_{c}^{ \pm}$to avoid the ultraviolet divergence. There are several prescriptions for the cutoff momenta depending on the physical problem for which the interaction is applied.

Prescription 1: This is the most simple prescription imposing on the single particle kinetic energy with the condition $\epsilon_{n}(k)<E_{c}$, i.e., $k_{c}^{+}=\sqrt{2 m^{*} E_{c}} / \hbar$ and $k_{c}^{-}=0$. It is independent of the Fermi momentum and of the pairing gap, but still has a weak dependence on the density through the effective mass $m^{*}(\rho)$. It has been used by several authors [14, 15, 16] and also adopted in shell model calculations in which all the shells up to a given cutoff energy are involved.

Prescription 2: This prescription is based on the fact that the pairing occurs among states around the Fermi energy. Then, it is natural to define the cutoff energy with respect to the Fermi momentum by the condition $\epsilon_{n}(k)<\epsilon_{F n}+E_{c}$, i.e., $k_{c}^{+}=\sqrt{2 m^{*}\left(\epsilon_{F n}+E_{c}\right)} / \hbar$ and still $k_{c}^{-}=0$ [25]. Through $\epsilon_{F}$, the dependence on the density of this cutoff is much stronger than for the prescription 1. This prescription is close to the prescription used in HFB calculations.

Prescription 3: This prescription is often used in the HFB calculations for which the cutoff is defined with respect to the quasi-particle energy $\sqrt{\left(\epsilon_{n}(k)-\nu_{n}\right)^{2}+\Delta_{n}^{2}}<E_{c}$. This leads to the following definition of the cutoff momenta: $k_{c}^{ \pm}=$ $\left[2 m^{*}\left(\nu_{n} \pm \sqrt{E_{c}^{2}-\Delta^{2}}\right)\right]^{1 / 2} / \hbar$ (if $E_{c}>\Delta_{n}$ ). If $k_{c}^{-}$becomes imaginary for very small $\nu_{n}$, we set $k_{c}^{-}=0$. In this prescription, the density dependence of the cutoff momenta $k_{c}^{ \pm}$is not trivial since it depends on the chemical potential and on the pairing gap. 


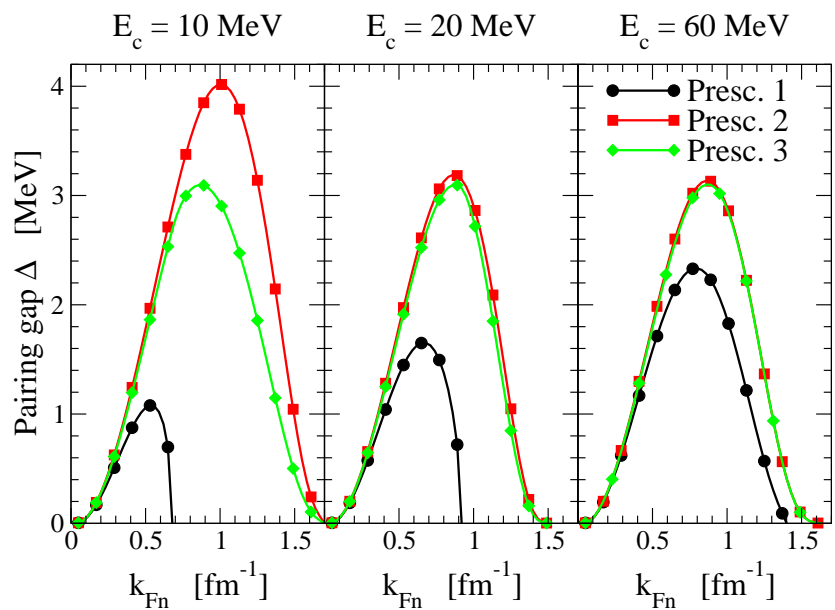

FIG. 10: (Color online) The pairing gap of symmetric nuclear matter as a function of the neutron Fermi momentum $k_{F n}$ for the 3 prescriptions. The solid, dashed, and dotted lines are for the prescriptions 1, 2, and 3, respectively. Each panel corresponds to a fixed value for the cutoff energy, $E_{c}=10,20$ or $60 \mathrm{MeV}$.

It should be noted that in the limit $E_{c} \gg \Delta_{n}$, the prescription 2 and 3 give the same cutoff momenta $k_{c}^{ \pm}$. If the limit $E_{c} \gg \epsilon_{F n}$ is also satisfied, then the three prescriptions are equivalent to each other. In Fig. 10 are represented the pairing gaps obtained in symmetric nuclear matter with the bare pairing interactions given in Table II. We compare the pairing gaps calculated for the three prescriptions with different values of the cutoff energy, 10, 20 and $60 \mathrm{MeV}$. For a low value of the cutoff energy $E_{c}=10 \mathrm{MeV}$, the three prescriptions lead to very different pairing gaps. For the cutoff energy larger than $20 \mathrm{MeV}$, the prescriptions 2 and 3 give very similar results because $E_{c} / \Delta_{n} \gg 1$. Notice that at very low Fermi momentum, $k_{F n}<0.4 \mathrm{fm}^{-1}\left(\rho_{n} / \rho_{0}<0.01\right)$, the three prescriptions give a similar pairing gap, because both conditions $E_{c} \gg \Delta_{n}$ and $E_{c} \gg \epsilon_{F n}$ are satisfied. Nevertheless, for $k_{F n}>0.4 \mathrm{fm}^{-1}$, the prescription 1 gives a pairing gap different from the prescriptions 2 and 3 , even for the larger cutoff energy $E_{c}=60 \mathrm{MeV}$. The reason is that the limit $E_{c} \gg \epsilon_{F n}$ is not reached for $k_{F n}>0.4 \mathrm{fm}^{-1}$.

\begin{tabular}{ccccc}
\hline \hline & \multicolumn{2}{c}{ Presc. 1 } & \multicolumn{2}{c}{ Presc. 2 } \\
$E_{c}[\mathrm{MeV}]$ & $\eta_{s}$ & $\alpha_{s}$ & $\eta_{s}$ & $\alpha_{s}$ \\
\hline 60 & 0.461 & 0.579 & 0.593 & 0.537 \\
40 & 0.413 & 0.487 & 0.657 & 0.506 \\
\hline \hline
\end{tabular}

TABLE V: Parameters of the density-dependent term $g=g_{1}$ in Eq. (1) obtained from the fit to the bare pairing gap in symmetric nuclear matter. The effective mass is obtained from SLy4 Skyrme interaction. The parameters for the prescription 3 are already shown in Table II
In Table $\nabla$ we give the parameters of the densitydependent term $g=g_{1}$ of Eq. (11) to fit the bare gap using the prescriptions 1 and 2 for symmetric nuclear matter. Those for the prescription 3 are already given in Table II It shows how sensible these parameters are on the cutoff prescription. One could remark that the parameter $\eta_{s}$ is much more affected by the prescription than the parameter $\alpha_{s}$. We can also compare our results with other calculations. The parameters obtained with the prescription 1 can be compared to the one proposed in Ref. [16], namely $\eta_{s}=0.45, \alpha_{s}=0.47$ for $E_{c}=60 \mathrm{MeV}$. The value of the parameter $\alpha_{s}$ is significantly different. One can nevertheless obtain a comparable value for the parameter $\alpha_{s}$ if one takes the approximation: $\nu_{n} \sim \epsilon_{F n}$. With the prescription 2 , we obtain similar parameters to those in Ref. [25] in which $\eta_{s}=0.60-0.63, \alpha_{s}=0.55-0.58$ are obtained. The small differences can be explained by a different effective mass and different adopted pairing gap.

\section{APPENDIX B: COOPER PAIR WAVE FUNCTION}

From the Cooper pair wave function (13) in the momentum space, we obtain the radial dependence of the Cooper pair wave function by a Fourier transform,

$$
\begin{aligned}
\Psi_{\text {pair }}(r) & =\frac{C}{(2 \pi)^{3}} \int d^{3} k u_{k} v_{k} e^{i \vec{k} \cdot \vec{r}} \\
& =C^{\prime} \int d k \frac{k^{2}}{E_{n}(k)} \frac{\sin k r}{k r}
\end{aligned}
$$

where the normalization constant $C^{\prime}$ is determined from the condition $\int d r r^{2}\left|\Psi_{\text {pair }}(r)\right|^{2}=1$. The wave function $\Psi_{\text {pair }}$ is separated into two terms $\Psi_{1}$ and $\Psi_{2}$ defined in Eqs. (16)-(17). The term $\Psi_{1}$ is solved numerically. If $k_{\infty} / k_{0} \gg 1$, with $k_{0}=\sqrt{2 m_{n}^{*} \nu_{n}} / \hbar$, we obtain the analytical form (18) for $\Psi_{2}(r)$ to the first order in $\nu_{n} / \epsilon_{n}(k)$, $1 / E_{n}(k) \sim 2 m_{n}^{*} / \hbar^{2} k^{2}$. It is shown in Fig. 11 that the convergence is very fast and $k_{\infty}=2 k_{0}$ provides already a good converged solution.

From the decomposition into $\Psi_{1}$ and $\Psi_{2}$ presented in Eq. (15), the treatment of Matsuo is equivalent to set $k_{\infty}=k_{c}$ and $\Psi_{2}=0$, where $k_{c}$ is the cutoff momentum according to the prescription 2 in Appendix A. By this treatment, a good agreement between the wave function obtained with the contact interaction and the one obtained with the Gogny interaction is reached for a cutoff energy $E_{c}=30 \mathrm{MeV}$. The agreement is very nice especially in the region $r<3 \mathrm{fm}$. This result is easily understood from the decomposition of $\Psi$ into $\Psi_{1}$ and $\Psi_{2}$. Namely, by introducing a cutoff energy in the definition of the Cooper pair wave function, Matsuo's modification effectively removes the singularity at $r \sim 0$. The justification of cutoff in the Cooper pair wave function could be understood from model space considerations. For practical reasons, the calculations for finite systems are never 

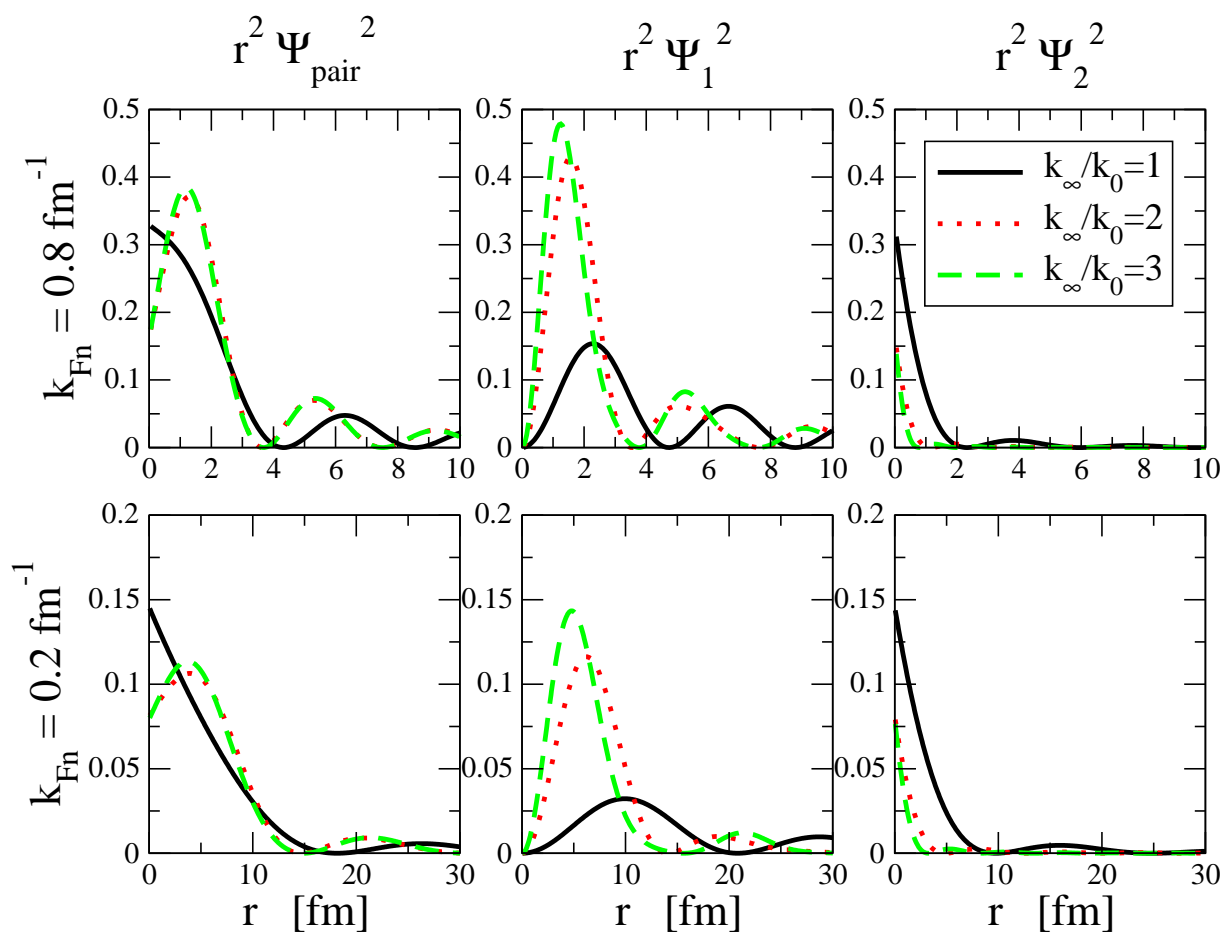

FIG. 11: (Color online) The Cooper pair wave function $r^{2} \Psi_{\text {pair }}^{2}$ as well as its individual contribution $r^{2} \Psi_{1}^{2}$ and $r^{2} \Psi_{2}^{2}$ for different values of the ratio $k_{\infty} / k_{0}$ and for two values of the neutron Fermi momentum. Notice that the convergence with respect to the ratio $k_{\infty} / k_{0}$ is very fast.

done with infinite basis but in a sub-basis involving a finite number of wave functions. A cutoff is then naturally introduced in the finite model space and all the quantities, including the Cooper pair wave function, are calculated within the same model space. The cutoff treatment of Matsuo is thus common in nuclear matter and finite nucleus calculations. However, it should be remarked that despite the singularity of the wave function in $\mathrm{nu}-$ clear matter, the rms radius $\xi_{r m s}$ stays finite and larger than $3 \mathrm{fm}$, in the range of explored densities. We have checked that the treatment of Matsuo only affects the rms radius by about $10 \%$ in the worst situation, i.e., it increases the rms radius by less than $0.5 \mathrm{fm}$ for neutron density at $\rho_{n} \sim \rho_{0} / 10$.
[1] M. Baldo, C. Maieron, P. Schuck and X. Viñas, Nucl.Phys. A736, 241 (2004).

[2] A. Sedrakian, T. T. S. Kuo, H. Müther, and P. Schuck, Phys. Lett. B576, 68 (2003).

[3] B.A. Brown, Phys. Rev. Lett 85, 5296 (2000).

[4] V. Baran and J. Margueron, Eur. Phys. J. A 30, 141 (2006); J. Margueron and Ph. Chomaz, Phys. Rev. C 71, 024318 (2005); V. Baran et al., Phys. Rev. Lett. 86, 4492 (2001).

[5] C.J. Horowitz, A. Schwenk, Nucl.Phys. A776, 55 (2006).

[6] A. Sedrakian and J. W. Clark, Phys. Rev. C 73, 035803 (2006).

[7] M. Baldo, U. Lombardo, and P. Schuck, Phys. Rev. C 52, 975 (1995).

[8] J. Margueron, E. van Dalen, and C. Fuchs, Phys. Rev. C 76, 034309 (2007).

[9] A. Bulgac, Phys. Rev. Lett. 95, 140403 (2005).

[10] U. Lombardo, in "Nuclear Methods and the Nuclear Equation of State", Int. Rev. of Nucl. Physics, Vol. 9,
M. Baldo Eds. (World-Scientific, Singapore, 1999).

[11] L. G. Cao, U. Lombardo, and P. Schuck, Phys. Rev. C 74, 064301 (2006).

[12] H. Heiselberg, C.J. Pethick, H. Smith, and L. Viverit, Phys. Rev. Lett. 85, 2418 (2000).

[13] H.-J. Schulze, A. Polls, and A. Ramos, Phys. Rev. C 63, 044310 (2001).

[14] G. F. Bertsch and H. Esbensen, Ann. Phys. (N.Y.) 209, 327 (1991).

[15] H. Esbensen and G. F. Bertsch, Nucl. Phys. A542, 310 (1992).

[16] H. Esbensen, G. F. Bertsch and K. Hencken, Phys. Rev. C 56, 3054 (1997).

[17] J. Dobaczewski, W. Nazarewicz, T. R. Werner, J. F. Berger, C. R. Chinn, and J. Dechargé, Phys. Rev. C 53, 2809 (1996).

[18] K. Hagino and H. Sagawa, Phys. Rev. C 72, 044321 (2005).

[19] N. Pillet, N. Sandulescu, and P. Schuck, Phys. Rev. C 
76, 024310 (2007).

[20] Ph. Chomaz, M. Colonna, J. Randrup, Phys. Rep. 389, 263 (2004).

[21] D. G. Yakovlev and C. J. Pethick, Annu. Rev. Astron. Astrophys. 42, 169 (2004).

[22] A. Sedrakian, Prog. Part. Nucl. Phys. 58, 168 (2007).

[23] Ch. Monrozeau, J. Margueron, and N. Sandulescu, Phys. Rev. C 75, 065807 (2007).

[24] K. Hagino, H. Sagawa, J. Carbonell, and P. Schuck, Phys. Rev. Lett. 99, 022506 (2007).

[25] M. Matsuo, Phys. Rev. C 73, 044309 (2006).

[26] A. Schwenk, B. Friman, and G.E. Brown, Nucl. Phys. A713, 191 (2003).

[27] A. Fabrocini, S. Fantoni, A. Yu. Illarionov, and K. E. Schmidt, Phys. Rev. Lett. 95, 192501 (2005).

[28] T. Abe, and R. Seki, arXiv:nucl-th/0708.2523.

[29] A. Gezerlis, and J. Carlson, arXiv:nucl-th/0711.3006.

[30] F. Barranco, R. A. Broglia, G. Gori, E. Vigezzi, P.-F. Bortignon and J. Terasaki, Phys. Rev. Lett. 83, 2147 (1999).

[31] N. Giovanardi, F. Barranco, R.A. Broglia, and E. Vigezzi, Phys. Rev. C 65, 041304(R) (2002).
[32] F. Barranco, P.F. Bortignon, R.A. Broglia, G. Colò, P. Schuck, E. Vigezzi, and X. Viñas, Phys. Rev. C 72, 054314 (2005).

[33] E. Garrido, P. Sarriguren, E. Moya de Guerra, and P. Schuck, Phys. Rev. C 60, 064312 (1999).

[34] Goriely et al., Nucl. Phys. A739, 331 (2004); Phys. Rev. C 66, 024326 (2002); Phys. Rev. C 68, 054325 (2003).

[35] J. Bardeen, L. N. Cooper, and J. R. Schrieffer, Phys. Rev. 108, 1175 (1957).

[36] P. G. de Gennes, Superconductivity of Metals and Alloys, (Addison-Wesley, Reading, MA, 1989).

[37] P. Ring and P. Schuck, The Nuclear Many-body Problem (Springer-Verlag, Heidelberg, 1980).

[38] Ph. Nozières and S. Schmitt-Rink, J. Low Temp. Phys. 59, 195 (1985).

[39] P. Pieri and G.C. Strinati, Phys. Rev. Lett., 91, 030401 (2003).

[40] J. R. Engelbrecht, M. Randeria, C. A. R. Sá de Melo, Phys. Rev B 55, 15153 (1997).

[41] T. Papenbrock and G. F. Bertsch, Phys. Rev. C 59, 2052 (1999). 